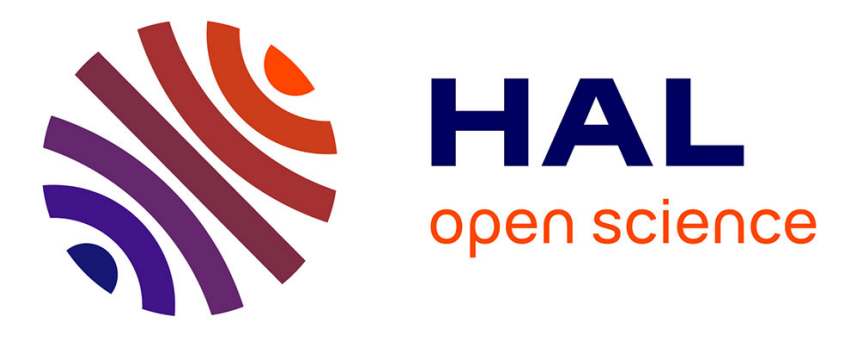

\title{
Ultrasound Testing of Metallic Structures using a Dual Symmetric Path Inspection and a Matched Filter-based Method
}

Costin Vasile, Petrut Teodor-Ion, Cornel Ioana, Valentin Sgârciu, Jerome I. Mars, Ion Candel

\section{To cite this version:}

Costin Vasile, Petrut Teodor-Ion, Cornel Ioana, Valentin Sgârciu, Jerome I. Mars, et al.. Ultrasound Testing of Metallic Structures using a Dual Symmetric Path Inspection and a Matched Filter-based Method. CAMSAP 2013 - 5th IEEE workshop on Computational Advances in Multi-Sensor Adaptive Processing, Dec 2013, Saint-Martin, France. pp.149-152, 10.1109/CAMSAP.2013.6714029 . hal00985502

\section{HAL Id: hal-00985502 https://hal.science/hal-00985502}

Submitted on 30 Apr 2014

HAL is a multi-disciplinary open access archive for the deposit and dissemination of scientific research documents, whether they are published or not. The documents may come from teaching and research institutions in France or abroad, or from public or private research centers.
L'archive ouverte pluridisciplinaire HAL, est destinée au dépôt et à la diffusion de documents scientifiques de niveau recherche, publiés ou non, émanant des établissements d'enseignement et de recherche français ou étrangers, des laboratoires publics ou privés. 


\title{
Ultrasound Testing of Metallic Structures using a Dual Symmetric Path Inspection and a Matched Filter-based Method
}

\author{
Costin Vasile $^{\# 1}$, Petrut Teodor-Ion ${ }^{\# 2}$, Cornel Ioana ${ }^{\# 3}$, Valentin Sgârciu ${ }^{\# 4}$, Jérôme Mars ${ }^{\# 5}$, Ion Candel ${ }^{\# 6}$ \\ \#2, \#3, \#5,\#6 Gipsa-lab, Grenoble Institute of Technology \\ 11 rue des Mathématiques, BP 46, Saint Martin d'Hères, 38402, France \\ ${ }^{2,3,5,6}$ \{ jerome.mars, teodor-ion.petrut, cornel.ioana, ion.candel\}@gipsa-lab.grenoble-inp.fr \\ ${ }^{\# 1, ~ \# 5}$ Faculty of Automatic Control and Computer Science, University Politehnica of Bucharest \\ 313 Splaiul Independentei, 060042, sector 6, Bucharest, ED 103-105 building, Romania \\ costin.vasile@aii.pub.ro \\ vsgarciu@aii.pub.ro
}

\begin{abstract}
In this paper, we present a new technique for detecting and localising defects in metallic structures, using ultrasound non-destructive inspection. This technique consists in applying a dual path inspection and the matched filter to a stepped frequency-type signal. This new technique is compared with classical non-destructive testing approaches. An experimental setup is presented that allows us to validate the proposed method.
\end{abstract}

\section{INTRODUCTION}

The detection and localisation of defects in metallic structures is a crucial aspect of any industrial endeavour, as any malfunction of a piece of equipment will impact production capabilities and will become the source of further spending in maintenance and repair. Thus, by taking preemptive actions to detect and localise defects in the earliest of stages, one can minimize these costs and the impact on production activities.

When using matched filtering, modulated excitation signals are commonly used in ultrasound inspection methods, due to the advantage of a filtering gain proportional to the bandwidth-duration product, with improvements of $12 \mathrm{~dB}$ to $18 \mathrm{~dB}$ for chirps [linear frequency modulation (FM)], or in the range of $4 \mathrm{~dB}$ to $9 \mathrm{~dB}$ for non-linear FM and binary codes [1], [2]. In this paper we propose to investigate the use of stepped frequency modulation in order to further exploit these advantages in the ultrasound inspection of metallic structures.

With this type of signal and the appropriate matched filter approach, we also introduced a symmetric path inspection method, which consists of inverting the roles of the two transducers that were used (the emitter becomes the receiver and vice versa), thus having two acquired received signals. These two signals will be repeatedly filtered with a timefrequency mask, which will then become the inputs for a matched filtering step in our proposed algorithm.

The blocks of steel on which the methods were tested present the worst case scenario for ultrasound testing: coarse grain structure, high attenuation and interference, longitudinal defects (parallel to the ultrasound waveform).

Section II will describe the two methods with further details (MF and MF-DSPI), in Section III we will present the experimental setup and results, followed by the conclusions in Section IV.

\section{MATCHED FILTERING (MF) AND DUAL SYMMETRIC PATH INSPECTION (MF-DSPI) METHODS}

\section{A. Matched Filtering (MF)}

Matched filtering is the preferred and most commonly used method for filtering signals with large bandwidth-duration product (such as linear frequency modulation, phase modulations, etc) and for identifying them in the presence of noise - for very low signal to noise ratios [3]. The matched filter is a linear time invariant filter, which maximizes the receiver SNR. Using the likelihood criterion, such a filter has the impulse response $h(t)$, equal to the time-reversed input waveform, $s(t),[1],[3]$ :

$$
h(t)=k \cdot s\left(\tau_{d}-t\right)
$$

,where $k$ is a gain factor and $\tau_{d}$ is the physical realisation generated time shift.

For the input $s(t)$, the matched filter output is given by equation (2):

$$
x(\tau)=k \cdot R_{s S}\left(\tau-\tau_{d}\right)
$$

,where $R_{s S}\left(\tau-\tau_{d}\right)$ is the time-shifted auto-correlation of the transmitted signal, $s(t)$. In other words, in our ultrasound application, the reception consists of several echoes, with the same form as the transmitted signals, but attenuated and corrupted by noise and false arrivals (interferences, reflections by grains, etc). A cross-correlation between the transmitted 
and the received signals will contain local maxima corresponding to the time of arrival of these echoes. In an ideal situation (in the presence of white noise), the matched filter output would be zero in the absence of echoes and would present maxima as the echoes arrive.

\section{B. Dual Symmetric Path Inspection (MF-DSPI)}

The basis of this approach is the symmetric path inspection of the steel block. This consists of two separate signal acquisitions, the first in which we send an excitation signal with one ultrasound transducer and receive with the other one, and the second where we inverse the roles of the transducers, where the emitter becomes the receiver and vice versa (figure 1). The reason behind this is that the two received signals will contain the useful echoes (backwall and defect generated echoes) while maintaining the same detection signal shape and the same time of arrival (TOA); the other echoes are "false alarms" (generated by metal impurities), with different properties (different TOA's and different detection signal signatures) due to their different relative positions to and from the transducers (thus, different refraction/reflection geometries). By analysing the two detection signals, we managed to greatly reduce the false alarm echoes, as we will show in the experimental results section.
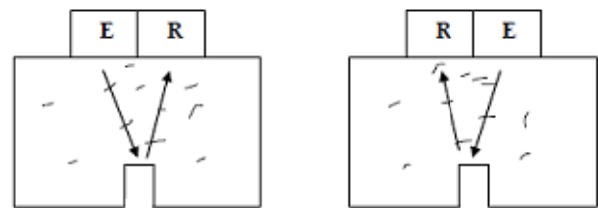

Fig. 1. The dual symmetric path inspection method.

The modulated excitation signal used was a stepped frequency modulation (a pseudo-chirp) (figure 2), with the following properties:

- 11 chirps, with a $30 \mathrm{KHz}$ bandwidth, centred at the following frequencies: $750 \mathrm{KHz}, 800 \mathrm{KHz}$, $850 \mathrm{KHz}, 900 \mathrm{KHz}, 950 \mathrm{KHz}, 1000 \mathrm{KHz}, 1050 \mathrm{KHz}$ $1100 \mathrm{KHz}, 1150 \mathrm{KHz}, 1200 \mathrm{KHz}, 1250 \mathrm{KHz}$;

- chirp duration: 15 microseconds; time between chirps: 100 microseconds; sampling frequency: $10 \mathrm{MHz}$

- entire signal repetition period: 13.1 milliseconds (enough time to avoid residual echoes, according to the block of steel used - figure 5);

- signals designed and adapted to the multiple scattering tendency of the material (experimentally - careful selection of frequency domain) [4].

An echo generated by a defect will have a smaller TOA than the TOA corresponding to the echo generated by the backwall of the steel block. It is therefore required to search for the excitation signal pattern only until right after the first backwall generated echo (including it, in order to always have a reference on the detection signals).

The excitation signal pattern was used for designing a timefrequency mask, that was applied on the received signals until right after the first backwall generated echo. Figures 2 and 3 show the time-frequency mask highlighted on the excitation signal and on a received signal.

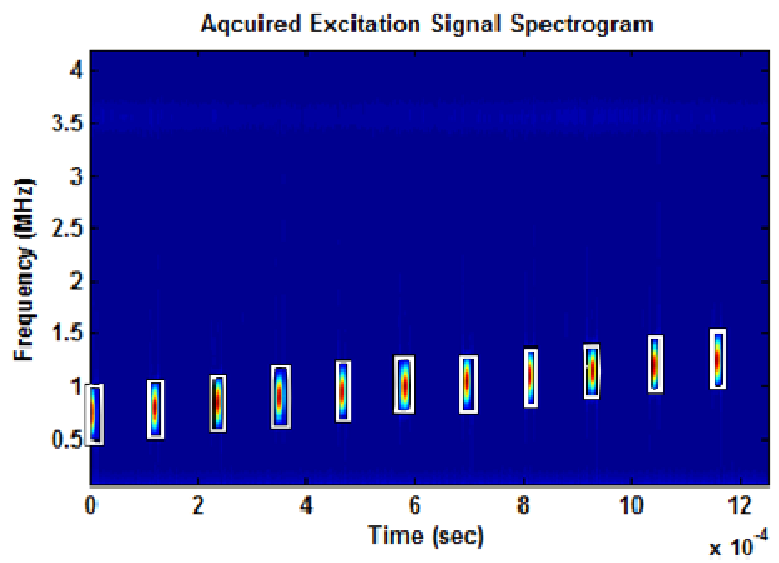

Fig. 2. Time-frequency mask built on the pattern of the excitation signal. (black rectangles around the 11 chirps).

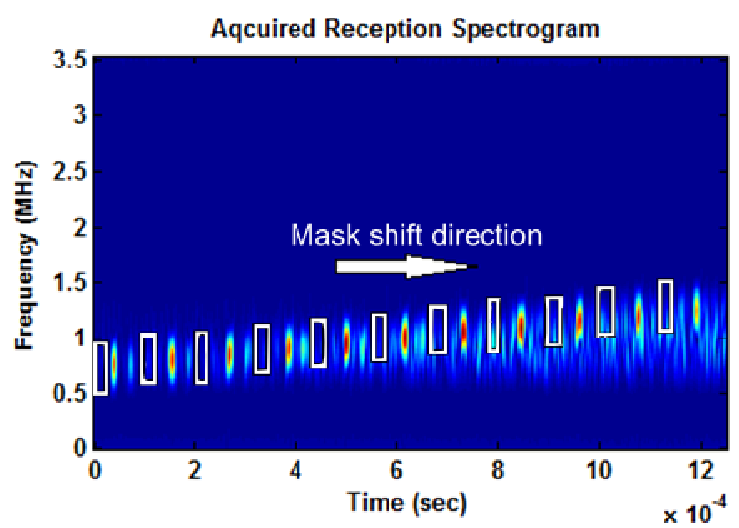

Fig. 3. Time-frequency mask applied on a received signal and direction of iterations.

The time-frequency mask was built in the following manner:

- time mask: selecting the time intervals corresponding to the excitation signal pattern;

- frequency mask: selecting the frequency intervals (Butterworth pass-band filters) corresponding to the bandwidths and centre frequencies of the 11 chirps that make up the excitation signal.

The time-frequency mask is applied only until right after the first backwall generated echo, as stated earlier, with an offset of 1 point between two consecutive applications. For each iteration, there is a matched filtering step, whose output will sum up to the previous iteration matched filtering step.

Thus, for iteration $i$, we have the filtered (in time-frequency domains) reception $m_{i}(t)$, and the previous iteration, $i-1$, matched filter output, $R_{\mathrm{i}-1}(\tau)$; the $i^{\text {th }}$ matched filter detection signal is given by:

$$
R_{i}(\tau)=R_{i-1}(\tau)+R_{m_{i}, s}(\tau)
$$


,where $s(t)$ is the pseudo-chirp excitation signal, and the initial detection signal is $R_{i=0}=0$. The final detection signals (for each of the two acquired signals: direct and inverse) will be $R_{i_{\text {final }}}^{d}(\tau)$ and $R_{i_{\text {final }}}^{i}(\tau)$, after the time-frequency and matched filtering steps.

The final step is to verify which portions of the two detection signals are consistent with one another, in order to detect and localise only the echoes corresponding to the faults in the metal block. These echoes must arrive at the same time and must have the same signatures, thus we have chosen to use, as a measure of verifying this, a sliding correlation coefficient ([5]) window, which should delete the vast majority of the false alarm echoes. This element-by-element sliding correlation coefficient window imposes the following criterion:

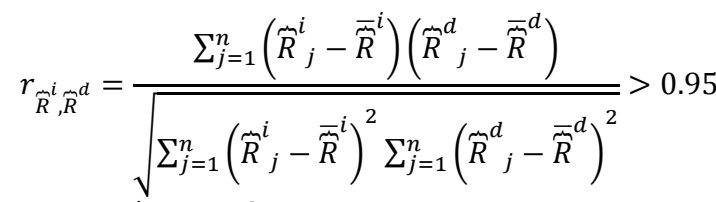

,where $\overline{\tilde{m}}^{i}$ and $\overline{\widetilde{M}}^{d}$ are the sample means of the sliding window as it advances through the two detection signals. By applying this criterion, we reduce the number of false alarm echoes and highlight the useful echoes. A block diagram for all the steps of the MF-DSPI method is presented in figure 4.

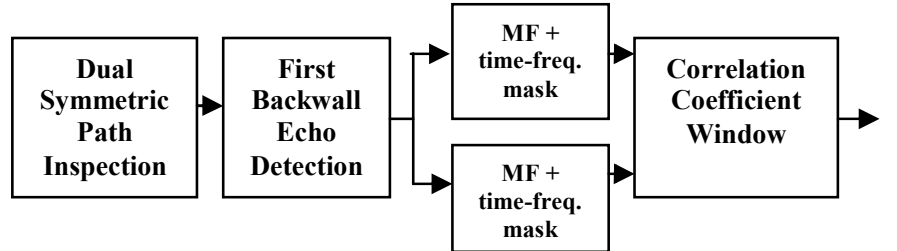

Fig. 4. Block diagram for the MF-DSPI method.

\section{EXPERIMENTAL SETUP AND RESULTS}

\section{A. Experimental Setup}

The central frequency of the ultrasound transducers is $1 \mathrm{MHz}$. The steel block that was used to verify and compare the two methods was a non-homogenous, low quality block of steel, with a coarse grain structure. Because of this, the attenuation of the ultrasound signals is higher, and the presence of randomly distributed scatterers introduced the increased rate of false alarms. Also, the defects which we tried to detect and localise were longitudinal, as seen in figure 5 (parallel to the ultrasound excitation signals), thus increasing the difficulty of the test case scenario.

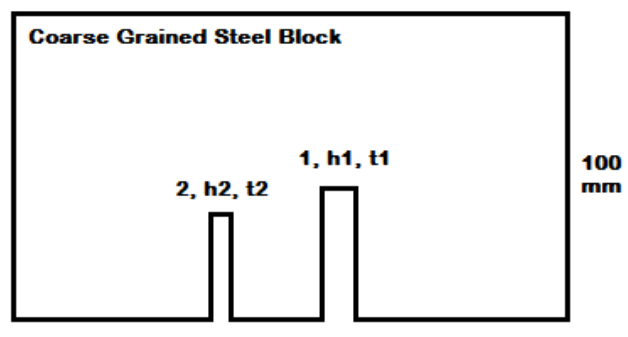

Fig. 5. Section view of the steel block.
The steel block has two defects (cavities), with the following properties (for the measured ultrasound velocity in the steel block of aprox. 5.52-5.53 millimetre/microsecond):

- Cavity 1 : height $h 1 \approx 30 \mathrm{~mm}$ and expected time $t 1 \approx 26$ microseconds;

- Cavity 2: height $h 2 \approx 25 \mathrm{~mm}$ and expected time $t 2 \approx 28.5$ microseconds.

\section{B. Results for Cavity 1}

Using the MF method, it is possible to detect the first (and largest) defect, followed by the backwall echo, as can be seen in figure 6 . The TOA of the echo is at 26 microseconds, which corresponds to the real position.

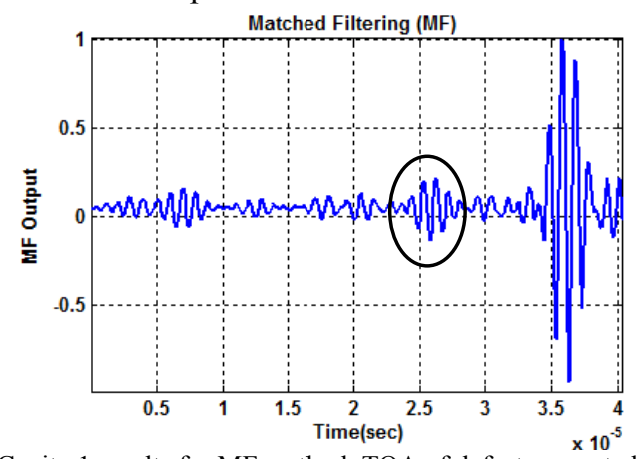

Fig. 6. Cavity 1 results for MF method. TOA of defect generated echo at 26 microseconds.

When applying dual path inspection and the MF with stepped frequency modulation the defect signature is enhanced at 26 microseconds (figure 7). In figure 8 we use the correlation coefficient criterion and the detection is very accurate.

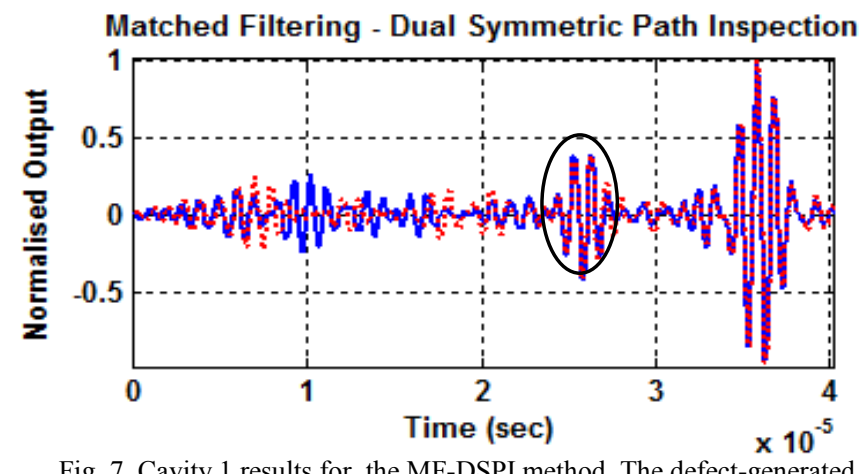

Fig. 7. Cavity 1 results for the MF-DSPI method. The defect-generated echo is much more visible (the two intermediate detection signals).

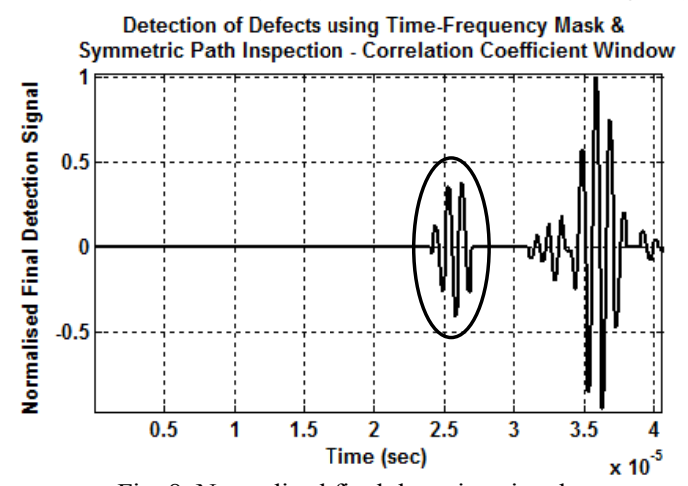

Fig. 8. Normalised final detection signal. 
The advantages of this method consist in amplifying the useful echoes and in the removal of the false alarm echoes.. In the case of the first cavity, it is clear how the defect-generated echo is not sufficiently highlighted when using the MF method, in comparison to the approach proposed in this paper. This is explained by the time-frequency mask that enables the precise search of the exact excitation signal pattern that was generated by the emitter.

\section{Results for Cavity 2}

When using the classic MF method for the second, smallest defect, we managed to find only the backwall generated echo, with no real sign of the cavity's presence. Figure 9 shows only the expected backwall generated echo, at 36 microseconds.

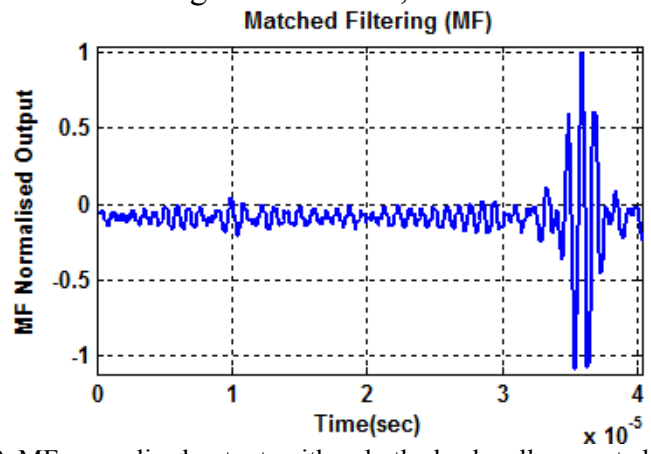

Fig. 9. MF normalised output, with only the backwall generated echo.

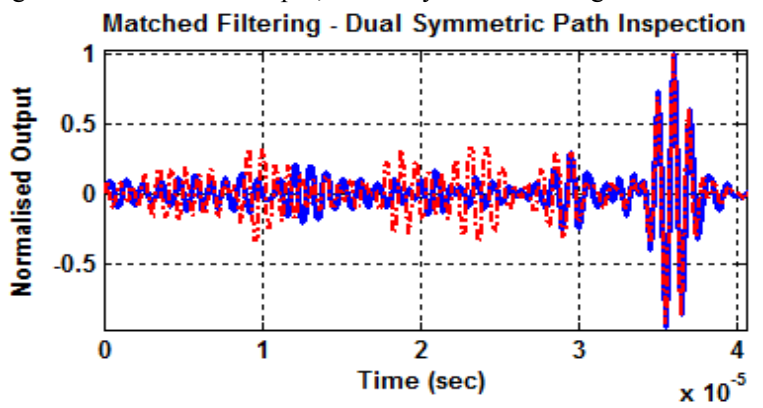

Fig. 10. MF-DSPI normalised output, with only backwall generated echo (the two intermediate detection signals).

The MF with stepped frequency modulation was also used, with similar results (figure 10): the matched filter gain is not sufficient to detect the echo coming from the cavity, because of the reduced reflected energy.

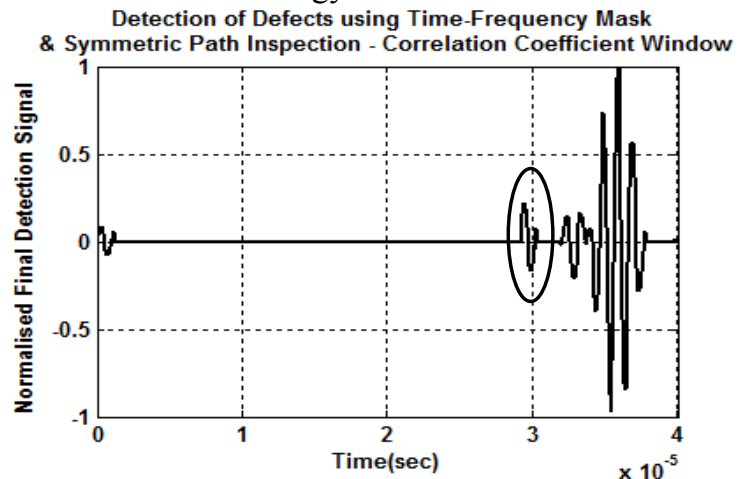

Fig. 11. Normalised Final Detection Signal, with the two useful echoes (defect generated echo at 29 microseconds).
However, when applying the sliding correlation coefficient window criterion, we discovered that the only similar portions of the two detection signals correspond the two useful echoes: the backwall (at 36 microseconds) and the defect generated echoes, detected at 29 microseconds, similar to the real position (figure 11).

\section{CONCLUSIONS}

This paper presents a comparison between the classical MF method and a new approach that is a combination of the dual symmetric path inspection and the matched filter, with also a stepped frequency modulation. We used these two methods to detect the useful echoes in the ultrasound inspection of a metallic steel block, and to minimize the impact of the false alarm echoes.

The improvements in terms of results are due to the following processing approaches: the symmetric path inspection exploits the similarity of useful echoes, the iterative time-frequency filtering of the received signal and its subsequent matched filtering and, lastly, the imposing of a criterion for similarity of useful echoes, consisting of a sliding correlation coefficient window.

The results show how the MF method is limited in a medium with high attenuation and in the presence of multiple false alarm-generating scatterers (coarse grain structure). The proposed approach succeeds in detecting and localising the defects, while also limiting the impact of these false alarm echoes. Also, choosing a stepped frequency modulated signal (and its frequency domain) aims to reduce multiple scattering by designing an appropriate time-frequency filter mask.

In the future works, we focus in the study of more advanced modulations and processing. The use of multielement transducer arrays is also another direction that will be pursued.

\section{REFERENCES}

[1] T. Misaridis and J.Jensen "Use of modulated excitation signals in medical ultrasound. part I: basic concepts and expected benefits," Ultrasonics, Ferroelectrics and Frequency Control, IEEE Transactions on, vol. 52, no. 2, pp. 177-191, 2005.

[2] T. Misaridis and J.Jensen "Use of modulated excitation signals in medical ultrasound. part II: design and performance for medical imaging applications," Ultrasonics, Ferroelectrics and Frequency Control, IEEE Transactions on, vol. 52, no. 2, pp. 192-205, 2005.

[3] S. Harput, JA Evans, N. Bubb and S. Freear "Diagnostic Ultrasound Tooth Imaging using Fractional Fourier Transform" Ultrasonics, Ferroelectrics and Frequency Control, IEEE Transactions, vol 58, no. 10, pp. 2096-2106, 2011.

[4] A. Aubry and A. Derode "Multiple scattering of ultrasound in weakly inhomogeneous media: Application to human soft tissues", Acoustical Society of America, vol 129, no. 1, pp. 225-233, 2011.

[5] J. Cornbleet and M. Shea "Comparison of Product Moment and Rank Correlation Coefficients in the Assessment of Laboratory MethodComparison Data", Clin.Chem, vol. 24, no. 6, pp. 857-861, 1978. 